PROCEEDINGS OF THE

AMERICAN MATHEMATICAL SOCIETY

Volume 128, Number 5, Pages 1467-1474

S 0002-9939(99)05153-9

Article electronically published on August 3, 1999

\title{
ON COMPACT MANIFOLDS WITH POSITIVE ISOTROPIC CURVATURE
}

\author{
M.-L. LABBI
}

(Communicated by Józef Dodziuk)

\begin{abstract}
In this paper we construct new Riemannian metrics with positive isotropic curvature on compact manifolds which fiber over the circle. We also study the relationship between the positivity of the isotropic curvature and the positivity of the $p$-curvature.
\end{abstract}

\section{InTRODUCTION AND STATEMENT OF RESUlTS}

Let $(M, g)$ be a Riemannian manifold of dimension $n$. For every $m \in M$ the inner product $g$ on the tangent space at $m, T_{m} M$, extends in two ways to the complexified tangent space $T_{m} M \otimes \mathbf{C}$ :

- as a complex bilinear form, to be denoted by $g(.,$.$) ,$

- as an Hermitian inner product, to be denoted by $\langle.,$.$\rangle .$

Let $\mathcal{R}: \wedge^{2} M \rightarrow \wedge^{2} M$ denote the curvature operator of $(M, g)$ and also its complex linear extension to $\wedge^{2} M \otimes \mathbf{C}$.

To each complex tangent two-plane $P \subset T_{m} M \otimes \mathbf{C}$ we assign its complex sectional curvature, denoted $K_{C}(P)$, which is a real number defined by

$$
K_{C}(P)=\frac{\langle\mathcal{R}(v \wedge w), v \wedge w\rangle}{\|v \wedge w\|^{2}}
$$

where $\{v, w\}$ is any basis of $P$.

A complex vector subspace $P \subset T_{m} M \otimes \mathbf{C}$ is called isotropic if $g(v, v)=0$ for all vectors $v \in P$.

Definition. A Riemannian manifold $(M, g)$ is said to have positive isotropic curvature if $K_{C}(P)>0$ for all two-dimensional isotropic subspaces of $T_{m} M \otimes \mathbf{C}$ and for every $m \in M$.

Now let $P$ be a complex two-plane and let $(z, w)$ be any basis of $P$. Note that the two-plane $P$ is isotropic if and only if we have

$$
g(z, z)=g(w, w)=g(z, w)=0 .
$$

If furthermore, the basis satisfies

$$
g(z, \bar{w})=0 \quad \text { and } \quad g(z, \bar{z})=g(w, \bar{w})=\sqrt{2}
$$

Received by the editors June 30, 1998.

1991 Mathematics Subject Classification. Primary 53B20, 53C21.

(C)2000 American Mathematical Society 
we call $(z, w)$ a standard basis of the isotropic two-plane $P$. In other words, $(z=$ $\left.e_{1}+i e_{2}, w=e_{3}+i e_{4}\right)$ is a standard basis for the isotropic plane $P$ if and only if $\left\{e_{1}, e_{2}, e_{3}, e_{4}\right\}$ are four orthonormal vectors in the real tangent space to $M$.

One can check easily from the definition that if $\left(z=e_{1}+i e_{2}, w=e_{3}+i e_{4}\right)$ is any standard basis for the isotropic plane $P$, then

$$
\begin{gathered}
K_{C}(P):=K_{C}(z, w)=K\left(e_{1}, e_{3}\right)+K\left(e_{1}, e_{4}\right)+K\left(e_{2}, e_{3}\right) \\
+K\left(e_{2}, e_{4}\right)-2 \mathcal{R}\left(e_{1}, e_{2}, e_{3}, e_{4}\right) .
\end{gathered}
$$

Now let $P_{1}$ (resp. $P_{2}$ ) denote the real two-plane associated to the complex vector $z$ (resp. $w$ ), that is, the real plane spanned by the real vectors $e_{1}$ and $e_{2}$ (resp. $e_{3}$ and $\left.e_{4}\right)$. Now if $\left(e_{1}^{\prime}, e_{2}^{\prime}\right)$ (resp. $\left.\left(e_{3}^{\prime}, e_{4}^{\prime}\right)\right)$ is any other orthonormal basis of $P_{1}$ (resp. $\left.P_{2}\right)$, then $\left(z^{\prime}=e_{1}^{\prime}+i e_{2}^{\prime}, w^{\prime}=e_{3}^{\prime}+i e_{4}^{\prime}\right)$ is also a standard basis for the same isotropic two-plane $P$. Thus, we obtain

$$
\begin{gathered}
K_{C}(P)=K_{C}(z, w)=K_{C}\left(z^{\prime}, w^{\prime}\right)=K\left(e_{1}^{\prime}, e_{3}^{\prime}\right)+K\left(e_{1}^{\prime}, e_{4}^{\prime}\right) \\
+K\left(e_{2}^{\prime}, e_{3}^{\prime}\right)+K\left(e_{2}^{\prime}, e_{4}^{\prime}\right)-2 \mathcal{R}\left(e_{1}^{\prime}, e_{2}^{\prime}, e_{3}^{\prime}, e_{4}^{\prime}\right) .
\end{gathered}
$$

This implies that in formula $(*)$, the isotropic curvature is independent from the choice of the orthonormal basis $\left(e_{1}, e_{2}\right)$ in $P_{1}$ and $\left(e_{3}, e_{4}\right)$ in $P_{2}$.

Hence one can write

$$
K_{C}(P)=K_{C}\left(P_{1}, P_{2}\right)
$$

where $\left(P_{1}, P_{2}\right)$ is a couple of orthogonal real two-planes in $P$ (seen as a real fourplane) associated to a standard basis $(z, w)$ of $P$ as above.

The existence of a metric with positive isotropic curvature on a compact manifold implies strong restrictions on its topology, indeed, Micallef and Moore [MM] proved the following:

Theorem. Let $M$ be a compact $n$-dimensional Riemannian manifold $(n \geq 4)$. If $M$ has positive isotropic curvature, then $\pi_{i}(M)=\{0\}$ for $2 \leq i \leq[n / 2]$. In particular, if $M$ is also simply connected, then $M$ is homeomorphic to a sphere.

Furthermore, Micallef and Wang [MW], and independently Seaman [Se], proved the following

Theorem. If $(M, g)$ is a compact Riemannian manifold of even dimension and with positive isotropic curvature, then $H^{2}(M, \mathbf{R})=0$.

On the other hand, little is known about examples of compact manifolds with positive isotropic curvature. In this context, Micallef and Wang [MW] proved the following interesting result:

Theorem. The connected sum of two manifolds of dimension $\geq 4$, each one admitting a metric with positive isotropic curvature, admits a metric with positive isotropic curvature.

In this paper, we construct new classes of manifolds with positive isotropic curvature. In Section 2, we study the relation between the positivity of the isotropic curvature and the positivity of the $p$-curvature. For example we prove that in the case of conformally flat manifolds the positivity of the $(n-4)$-curvature $(n$ is the dimension of the manifold) is equivalent to the positivity of the isotropic curvature, and we deduce many consequences.

In Sections 3 and 4, we prove the following theorems: 
Theorem I. Let $\pi:(M, g) \rightarrow S^{1}$ be a local Riemannian submersion over the circle, where $M$ is a compact Riemannian manifold of dimension $\geq 5$.

If the fibers of $\pi$ (endowed with the induced metric) satisfy the positivity condition (A), then the manifold $M$ admits a metric with positive isotropic curvature, where (A) denotes the following positivity condition for an algebraic curvature tensor:

For all orthonormal vectors $e_{i}, e_{j}, e_{k}, e_{l}$, we have

$$
K\left(e_{j}, e_{k}\right)+K\left(e_{j}, e_{l}\right)>\left|\mathcal{R}\left(e_{i}, e_{j}, e_{k}, e_{l}\right)\right|
$$

where $K$ is its sectional curvature.

Note that the strict pointwise $\frac{1}{4}$-pinching condition implies the condition (A), and the condition (A) implies at the same moment the positivity of the isotropic curvature and the positivity of the Ricci curvature. Note also that in the case of conformally flat manifolds and hypersurfaces of the Euclidean space, it is equivalent to the following positivity condition on the sectional curvature:

$$
K\left(e_{j}, e_{k}\right)+K\left(e_{j}, e_{l}\right)>0, \quad \text { for all orthonormal vectors } e_{j}, e_{k}, e_{l} .
$$

In the case of four dimensions, we prove the following similar result:

Theorem II. Let $\pi:(M, g) \rightarrow S^{1}$ be a Riemannian submersion over the circle, where $M$ is a compact Riemannian manifold of dimension 4.

If the fibers of $\pi$ (endowed with the induced metric) are with positive Ricci curvature, then the manifold $M$ admits a metric with positive isotropic curvature.

Example. Let $F$ be any manifold of $\operatorname{dim} \geq 3$ admitting a metric which satisfies the positivity condition (A) (if $\operatorname{dim} F=3$, we suppose that $F$ is with positive Ricci curvature), and let $\phi \in I \operatorname{som}(F)$ and define

$$
\begin{gathered}
\rho: \mathbf{Z} \longrightarrow \operatorname{Isom}(F \times \mathbf{R}) \\
n \longrightarrow \phi_{n}(x, t)=\left(\phi^{n}(x), t+n\right) .
\end{gathered}
$$

The manifold $M=\frac{F \times \mathbf{R}}{\rho}$ is the total space of a Riemannian submersion and satisfies the conditions of Theorem I or II, hence it admits a Riemannian metric with positive isotropic curvature.

As a direct consequence of the proofs of the previous Theorems I and II, we can easily prove the following corollaries:

Corollary I. If a compact manifold of dimension $\geq 5$ (resp. of dimension 4) admits a Riemannian foliation of codimension 1 such that the leaves, endowed with the induced metric, satisfy the positivity condition (A) (resp. are with positive Ricci curvature), then it admits a Riemannian metric with positive isotropic curvature.

Corollary II. If a compact manifold $M$ of dimension 4 admits a free action of $S U(2)$ or $S O(3)$, then it admits a Riemannian metric with positive isotropic curvature.

Remarks. 1. This last theorem is not generally true if the action is not supposed to be free. In fact, $S^{2} \times S^{2}$ admits an effective action of $S O(3)$, but no metric with positive isotropic curvature since the second Betti number is not zero.

2. One can immediately obtain from our previous results and the results of Micallef-Moore and Wang well known topological obstructions (such as the annulation of certain homotopy and homology groups) on the existence of Riemannian foliations and group actions on compact manifolds. 


\section{Positive isotropic CuRvature And positive $p$-Curvature}

Let $(M, g)$ be a Riemannian manifold of dimension $n$. Recall that (see [L1], [L2] and [L3]) the $p$-curvature of $(M, g)$, denoted by $s_{p}, 0 \leq p \leq n-2$, is a function defined on the $p$-Grassmannian bundle over $M$, which assigns to every tangent $p$-plan $P$ at $m \in M$ the scalar curvature at $m$ of the Riemannian sub-manifold $\exp _{m}(V)$, where $V$ is a neighborhood of 0 in $P^{\perp}$ (the orthogonal to $P$ in $T_{m} M$ ), and $\exp _{m}$ is the exponential map.

For $p=0$ (resp. $p=n-2$ ) it is the scalar curvature (resp. the sectional curvature). Note that the positivity of $s_{p}$ implies the positivity of $s_{k}$ for all $k=$ $0, \ldots, p-1$.

Proposition. Let $(M, g)$ be an $n$-dimensional Riemannian manifold with positive (resp. nonnegative) isotropic curvature. Then it has positive (resp. nonnegative) p-curvature, for all $p \leq n-4$.

Proof. From the formula $(*)$ stated in the introduction, we have

$$
\begin{aligned}
& K\left(e_{1}, e_{3}\right)+K\left(e_{1}, e_{4}\right)+K\left(e_{2}, e_{3}\right)+K\left(e_{2}, e_{4}\right)-2 \mathcal{R}\left(e_{1}, e_{2}, e_{3}, e_{4}\right)>0, \\
& K\left(e_{1}, e_{4}\right)+K\left(e_{1}, e_{3}\right)+K\left(e_{2}, e_{4}\right)+K\left(e_{2}, e_{3}\right)+2 \mathcal{R}\left(e_{1}, e_{2}, e_{3}, e_{4}\right)>0
\end{aligned}
$$

for all orthonormal tangent vectors $\left(e_{1}, e_{2}, e_{3}, e_{4}\right)$.

It follows that

$$
K\left(e_{1}, e_{3}\right)+K\left(e_{1}, e_{4}\right)+K\left(e_{2}, e_{3}\right)+K\left(e_{2}, e_{4}\right)>0 .
$$

By permuting the indices, one can easily check that this implies

$$
K\left(e_{1}, e_{2}\right)+K\left(e_{1}, e_{3}\right)+K\left(e_{1}, e_{4}\right)+K\left(e_{2}, e_{3}\right)+K\left(e_{2}, e_{4}\right)+K\left(e_{3}, e_{4}\right)>0
$$

for all orthonormal tangent vectors $\left(e_{1}, e_{2}, e_{3}, e_{4}\right)$, which is nothing but the positivity of the $(n-4)$-curvature. Consequently, the $p$-curvature is positive, for all $p \leq n-4$.

Of course we obtain large inequality in the case of nonnegative curvature.

Remark. Note that the converse, in the previous proposition, is not generally true. In fact, the product of a sphere of dimension $n-2$ with a Riemann surface of genus $g \geq 2$ admits a metric with positive $(n-4)$-curvature (see [L2]), but no metric with positive isotropic curvature, since the second Betti number is not zero.

In the case when $n=4$, Micallef and Moore (see [MM] and [MWo]) proved that $(M, g)$ has positive isotropic curvature if and only if $\frac{1}{6} s_{0}-W>0$ as an operator on $\wedge^{2} M$, where $s_{0}$ is the scalar curvature and $W$ is the Weil curvature. We can generalize this result to any dimension as follows:

Proposition. A Riemannian manifold $(M, g)$ has positive isotropic curvature if and only if for all four-planes $P$ tangent to $M$ we have

$$
\frac{1}{6} s_{n-4}\left(P^{\perp}\right)-W>0
$$

as an operator on $\wedge^{2} P$, where $s_{n-4}\left(P^{\perp}\right)$ is the $(n-4)$-curvature of $(M, g)$ and $W: \wedge^{2} P \rightarrow \wedge^{2} P$ is the Weyl curvature operator at $m$ of the Riemannian submanifold $\exp _{m}(V)$, where $V$ is a neighborhood of 0 in $P$.

Proof. This follows immediately from the case $n=4$ after noting that the Riemannian submanifold $\exp _{m}(V)$ is totally geodesic at $m$ and its dimension is four. 
Corollary I. Let $(M, g)$ be a compact conformally flat $n$-manifold with positive isotropic curvature. Then $H^{m}(M, \mathbf{R})=0$ for $2 \leq m \leq n-2$.

Corollary II. Let $(M, g)$ be a compact conformally flat $n$-manifold with nonnegative isotropic curvature such that $H^{2}(M, \mathbf{R}) \neq 0$. Then $(M, g)$ is either flat or covered by $S^{n-2} \times H^{2}$.

Proof. In the case of conformally flat manifolds, the Weil curvature is zero. Consequently, the positivity of the $(n-4)$-curvature is equivalent to the positivity of the isotropic curvature, and hence, the corollaries are immediate consequences of our similar results on the $p$-curvature in [L1].

This generalizes the result of Micallef and Wang $[\mathrm{MW}]$ in the case of conformally flat manifolds. This was first proved by S. Nayatani in $[\mathrm{Na}]$.

\section{Proofs of Theorems I AND II}

Let $\pi:(M, g) \rightarrow S^{1}$ be a Riemannian submersion. For all $m \in M$, the tangent space $T_{m} M$ decomposes into

$$
T_{m} M=\mathcal{V}_{m} \oplus \mathbf{R} \tau
$$

where $\mathcal{V}_{m}$ is the subspace of $T_{m} M$ tangent to the fiber at $m$ (called the vertical subspace), and $\tau$ is the unit vector orthogonal to $\mathcal{V}_{m}$.

Now let $g_{t}, t \in \mathbf{R}$, be the canonical variation of the metric $g$, that is, a new Riemannian metric defined on $M$ by (see [Be] and [L2])

$$
\begin{gathered}
g_{t \mid \mathcal{V}_{m}}=t^{2} g_{\mid \mathcal{V}_{m}}, \\
g_{t}(\tau, \tau)=g(\tau, \tau), \\
g_{t}\left(\mathcal{V}_{m}, \tau\right)=0 .
\end{gathered}
$$

Using the O'Neill formulas for the curvature, one can prove without difficulties the following (see [L2], [Be]).

Lemma. Let $R_{t}$ denote the curvature tensor for the metric $g_{t}$. For all $g_{t}$-unit vectors $U_{i}$ in $\mathcal{V}_{m}$, we have

$$
\begin{gathered}
R_{t}\left(U_{1}, U_{2}, U_{3}, U_{4}\right)=\frac{1}{t^{2}} \hat{R}\left(t U_{1}, t U_{2}, t U_{3}, t U_{4}\right)+O\left(\frac{1}{t}\right), \\
R_{t}\left(U_{1}, U_{2}, U_{3}, \tau\right)=O\left(\frac{1}{t}\right), \\
R_{t}\left(U_{1}, \tau, U_{2}, \tau\right)=O(1),
\end{gathered}
$$

where $\hat{R}$ is the curvature tensor for the induced metric on the fibers from the metric $g$.

Now let $P$ be any isotropic two-plane in $\left(T_{m} M \otimes \mathbf{C}, g_{t}\right)$; and let $\left(P_{1}, P_{2}\right)$ be a couple of orthogonal real two-planes associated to a standard basis of $P$ (as mentioned in the introduction).

Since $\operatorname{dim} \mathcal{V}_{m}=n-1 \geq 3$, and since the isotropic curvature is independent from the choice of the standard basis in $P$, we can assume that $P_{1}$ or $P_{2}$ is contained in $\mathcal{V}_{m}$ (see Lemma below). Suppose for example that $P_{2} \subset \mathcal{V}_{m}$, and then let $\left(U_{2}, V_{2}\right)$ be any $g_{t}$-orthonormal basis for $P_{2}$, such that the vectors $U_{2}$ and $V_{2}$ are in $\mathcal{V}_{m}$.

Since now $\operatorname{dim}\left(P_{1} \cap \mathcal{V}_{m}\right) \geq 1$, let $U_{1}$ be a $g_{t}$-unit vector in $P_{1} \cap \mathcal{V}_{m}$ and $E_{1}$ be a $g_{t}$-unit vector orthogonal to $U_{1}$ in $P_{1}$. Set $E_{1}=\alpha_{1} \tau+\beta_{1} V_{1}$, where $V_{1}$ is a $g_{t}$-unit vector in $\mathcal{V}_{m}$. Thus $\left(U_{1}, E_{1}\right)$ is a $g_{t}$-orthonormal basis for $P_{1}$. 
The isotropic curvature of the two-isotropic plane $P$ for the metric $g_{t}$ is then given by

$$
\begin{aligned}
\operatorname{Isot}_{t}\left(P_{1}, P_{2}\right)= & K_{t}\left(U_{1}, U_{2}\right)+K_{t}\left(U_{1}, V_{2}\right)+K_{t}\left(E_{1}, U_{2}\right) \\
& +K_{t}\left(E_{1}, V_{2}\right)-2 R_{t}\left(U_{1}, E_{1}, U_{2}, V_{2}\right)
\end{aligned}
$$

Using the previous Lemma, we obtain

$$
\begin{gathered}
\operatorname{Isot}_{t}(P)=\frac{1}{t^{2}} \hat{K}\left(U_{1}^{1}, U_{2}^{1}\right)+\frac{1}{t^{2}} \hat{K}\left(U_{1}^{1}, V_{2}^{1}\right)+\frac{\beta_{1}^{2}}{t^{2}} \hat{K}\left(V_{1}^{1}, U_{2}^{1}\right)+\frac{\beta_{1}^{2}}{t^{2}} \hat{K}\left(V_{1}^{1}, V_{2}^{1}\right) \\
-\frac{2 \beta_{1}}{t^{2}} \hat{R}\left(U_{1}^{1}, V_{1}^{1}, U_{2}^{1}, V_{2}^{1}\right)+O\left(\frac{1}{t}\right)
\end{gathered}
$$

where $U_{i}^{1}=t U_{i}$ and $V_{1}^{1}=t V_{i}$ are $g$-unit vectors in $\mathcal{V}_{m}$. It follows that

$$
\operatorname{Isot}_{t}(P) \geq \frac{1}{t^{2}} F\left(\beta_{1}\right)+O\left(\frac{1}{t}\right)
$$

where

$$
\begin{aligned}
F\left(\beta_{1}\right)= & \hat{K}\left(U_{1}^{1}, U_{2}^{1}\right)+\hat{K}\left(U_{1}^{1}, V_{2}^{1}\right)+\beta_{1}^{2} \hat{K}\left(V_{1}^{1}, U_{2}^{1}\right)+\beta_{1}^{2} \hat{K}\left(V_{1}^{1}, V_{2}^{1}\right) \\
& -2\left|\beta_{1}\right|\left|\hat{R}\left(U_{1}^{1}, V_{1}^{1}, U_{2}^{1}, V_{2}^{1}\right)\right|
\end{aligned}
$$

Hence, since $M$ is compact, the isotropic curvature $I$ sot $_{t}$ will be positive on all $M$ for $t$ small enough, if we have $F\left(\beta_{1}\right)>0$ for all $0 \leq \beta_{1} \leq 1$.

Let us now look for the positivity of this function. We have

$$
\begin{gathered}
F(0)=\hat{K}\left(U_{1}^{1}, U_{2}^{1}\right)+\hat{K}\left(U_{1}^{1}, V_{2}^{1}\right)>0, F^{\prime}(0)=-2\left|\hat{R}\left(U_{1}^{1}, V_{1}^{1}, U_{2}^{1}, V_{2}^{1}\right)\right| \leq 0 \\
F(1)=\operatorname{Isot}\left(\hat{P}_{1}, \hat{P}_{2}\right)>0 \\
F^{\prime}(1)=2\left(\hat{K}\left(V_{1}^{1}, U_{2}^{1}\right)+\hat{K}\left(V_{1}^{1}, V_{2}^{1}\right)\right)-2\left|\hat{R}\left(U_{1}^{1}, V_{1}^{1}, U_{2}^{1}, V_{2}^{1}\right)\right| .
\end{gathered}
$$

If $F^{\prime}(1) \leq 0$, then $F$ will decrease and then $F(1)>0$ implies that $F$ is positive $\forall \beta, 0 \leq \beta \leq 1$.

Now, if $F^{\prime}(1)>0$, that is, $\hat{K}\left(V_{1}^{1}, U_{2}^{1}\right)+\hat{K}\left(V_{1}^{1}, V_{2}^{1}\right)>\left|\hat{R}\left(U_{1}^{1}, V_{1}^{1}, U_{2}^{1}, V_{2}^{1}\right)\right|$, then the function $F$ admits a minimum value at

$$
\beta=\frac{\left|\hat{R}\left(U_{1}^{1}, V_{1}^{1}, U_{2}^{1}, V_{2}^{1}\right)\right|}{\hat{K}\left(V_{1}^{1}, U_{2}^{1}\right)+\hat{K}\left(V_{1}^{1}, V_{2}^{1}\right)}
$$

Its value is equal to

$$
\hat{K}\left(V_{1}^{1}, U_{2}^{1}\right)+\hat{K}\left(V_{1}^{1}, V_{2}^{1}\right)-\frac{\left|\hat{R}\left(U_{1}^{1}, V_{1}^{1}, U_{2}^{1}, V_{2}^{1}\right)^{2}\right|}{\hat{K}\left(V_{1}^{1}, U_{2}^{1}\right)+\hat{K}\left(V_{1}^{1}, V_{2}^{1}\right)} .
$$

This value is positive if $\hat{K}\left(V_{1}^{1}, U_{2}^{1}\right)+\hat{K}\left(V_{1}^{1}, V_{2}^{1}\right)>\left|\hat{R}\left(U_{1}^{1}, V_{1}^{1}, U_{2}^{1}, V_{2}^{1}\right)\right|$. This is nothing but the condition (A) in our theorem.

Lemma. Let $T_{m} M=\mathcal{V}_{m} \oplus \mathbf{R} \tau$ as above, where $\operatorname{dim} \mathcal{V}_{m}=n-1$. Then every isotropic two-plane $P$ in $\left(T_{m} M \otimes \mathbf{C}, g_{t}\right)$ has a standard basis $\left(z=U_{1}+i V_{1}, w=\right.$ $\left.U_{2}+i E_{2}\right)$, such that the vectors $U_{1}, V_{1}$ and $U_{2}$ are in $\mathcal{V}_{m}$.

Proof. Let $\left(z=U_{1}+i E_{1}, w=U_{2}+i E_{2}\right)$ be any standard basis for $P$, and let $\left(P_{1}, P_{2}\right)$ be the associated couple of orthogonal real two-planes as in the introduction.

Since $\operatorname{dim}\left(P_{i} \cap \mathcal{V}_{m}\right) \geq 1$ for $i=1,2$, we can assume that $U_{1}$ and $U_{2}$ are in $\mathcal{V}_{m}$. 
Now, using a rotation in $T_{m} M \otimes \mathbf{C}$, one can suppose that $E_{1}$ is also in $\mathcal{V}_{m}$. In fact, set $\alpha_{i}=g_{t}\left(\tau, E_{i}\right)$ for $i=1,2$. If $\alpha_{1}=0$, then $E_{1} \in \mathcal{V}_{m}$. Suppose $\alpha_{1} \neq 0$; then one can check easily that

$$
\begin{aligned}
& \left(z^{\prime}=\frac{\alpha_{1}^{2}}{\alpha_{1}^{2}+\alpha_{2}^{2}}\left(\left(U_{2}+i E_{2}\right)-\frac{\alpha_{2}}{\alpha_{1}}\left(U_{1}+i E_{1}\right)\right)\right. \\
& \left.w^{\prime}=\frac{\alpha_{1}^{2}}{\alpha_{1}^{2}+\alpha_{2}^{2}}\left(\frac{\alpha_{2}}{\alpha_{1}}\left(U_{2}+i E_{2}\right)+\left(U_{1}+i E_{1}\right)\right)\right)
\end{aligned}
$$

is also a standard basis for $P$.

Then, one can write this standard basis in the form $\left(z^{\prime}=U_{1}^{\prime}+i E_{1}^{\prime}, w^{\prime}=U_{2}^{\prime}+i E_{2}^{\prime}\right)$, where

$$
\begin{aligned}
& U_{1}^{\prime}=\frac{\alpha_{1}^{2}}{\alpha_{1}^{2}+\alpha_{2}^{2}}\left[U_{2}-\frac{\alpha_{2}}{\alpha_{1}} U_{1}\right] \in \mathcal{V}_{m}, \\
& E_{1}^{\prime}=\frac{\alpha_{1}^{2}}{\alpha_{1}^{2}+\alpha_{2}^{2}}\left[E_{2}-\frac{\alpha_{2}}{\alpha_{1}} E_{1}\right] \in \mathcal{V}_{m}, \\
& U_{2}^{\prime}=\frac{\alpha_{1}^{2}}{\alpha_{1}^{2}+\alpha_{2}^{2}}\left[U_{1}+\frac{\alpha_{2}}{\alpha_{1}} U_{2}\right] \in \mathcal{V}_{m},
\end{aligned}
$$

as required by the lemma.

This completes the proof of Theorem I.

The proof of Theorem II is similar. It suffices to remark that in the case when the dimension of $M$ is 4 , we must have in the previous proof $E_{1}= \pm \tau$, that is, $\beta_{1}=0$, since $E_{1}$ and $\tau$ are at the same moment orthogonal to $U_{1}, U_{2}, V_{2}$. Then Isot $_{t}$ will be positive on $M$ if $F(0)$ is positive, which is nothing but the positivity of the Ricci curvature. This completes the proof of Theorem II.

\section{Proofs of the COROLlaries}

Proof of Corollary I. If the manifold admits a Riemannian foliation, then it is locally the total space of a Riemannian submersion. It results then immediately from the previous proofs of Theorems I and II, that such a manifold admits a Riemannian metric with positive isotropic curvature.

Proof of Corollary II. Since the action of the group $G$ on the manifold $M$ is free, the canonical projection $M \rightarrow M / G$ is a smooth submersion ( $G$ denotes $S U(2)$ or $S O(3))$. Let us equip the fibers with a biinvariant metric of $G$ by using the natural inclusion $\mathcal{G} \hookrightarrow T_{m} M$.

On the other hand, using a $G$-invariant metric on $M$, we define the horizontal distribution and we lift it to a fixed Riemannian metric from $M / G$. Hence the projection $M \rightarrow M / G$ is a Riemannian submersion such that its fibers satisfy the condition of Theorem II. Hence, it admits a Riemannian metric with positive isotropic curvature.

Final remarks. 1. Note that the class of manifolds given by Theorems I and II does not admit any metric with positive Ricci curvature since the first Betti number is not zero, in fact the pull-back of the standard form on the circle to such manifolds is a nonzero closed 1-form.

2. We proved in [L3] that the positivity of the $(n-4)$-curvature is stable under surgeries in codimension $\geq n-1$ ( $n$ is the dimension of the manifold). Since it is close to the positivity of the isotropic curvature, one can think that a similar result 
will be true for positive isotropic curvature. Note that this is true in codimension $n$ since a surgery in codimension $n$ is a connected sum (this is the result of [MW]). This remark was pointed out to Gromov by Mario Micallef (see [Gr]). But unfortunately an adaptation of our proof in the case of the $p$-curvature [L3] to the case of isotropic curvature is possible only when the codimension is $n$ (and not $n-1$ ).

\section{ACKNOWLEDGEMENTS}

I would like to thank Professor J. Lafontaine for useful discussions, the ICTP (Trieste-Italy) for its help during this work and the Mathematics Department of the University of Bahrain where part of this research was done.

\section{REFERENCES}

[Be] Besse A. L., Einstein manifolds, Springer-Verlag (1987). MR 88f:53087

[Gr] Gromov M., Positive curvature, macroscopic dimension, spectral gaps and higher signatures, Preprint I.H.E.S.

[L1] Labbi M. L., Sur les nombres de Betti des variétés conformément plates, C. R. Acad. Sci. Paris, t. 319, série I, 77-80 (1994). MR 95f:53075

[L2] LabBi M. L., Actions des groupes de Lie presques simples et positivité de la $p$-courbure, Annales de la faculte des sciences de Toulouse, vol5. n2. (1997) 263-276. (France). CMP 98:09

[L3] LABBi M. L., Stability of the $p$-curvature positivity under surgeries and manifolds with positive Einstein tensor, Annals of Global Analysis and Geometry, August (1997). MR 98e:53065

[LY] LAwSON H.B., YAu S.T., Scalar curvature, non abelian group actions, and the degree of symmetry of exotic spheres, Comment.Math.Helv.49.(1974) 232-244. MR 50:11300

[MM] Micallef M. J., Moore J. D., Minimal two spheres and the topology of manifolds with positive curvature on totally isotropic two planes, Annals of Mathematics, 127(1988), 199-227. MR 89e:53088

[MW] Micallef M.J., Wang M.Y., Metrics with nonegative isotropic curvature, Duke Mathematic Journal, vol72. n3.(1993) 649-672 MR 94k:53052

[MWo] Micallef M.J., Wolfson J.G., The second variation of area of minimal surfaces in four manifolds, Math. Ann. 295(1993) 245-267 MR 94c:58035

[Na] Nayatani, S., Kleinian groups and conformally flat metrics, Proceedings of the First MSJ International Research Institute on Geometry and Global Analysis, July 12-23, (1993), Tohuku University, Sendai, Japan. MR 98a:53059

[Se] SEAman W., On manifolds with nonegative curvature on totally isotropic 2-planes, Transactions of the American Mathematical Society, volume 338, n2,843-855(1993). MR 93j:53053

Department of mathematics, College of Sciences, University of Bahrain, P.O. Box 32038, ISA TOWN, BAHRAIN

E-mail address: labbi@sci.uob.bh

Permanent address: 4, Rue de Sicile, Les Bioclimatiques n4, 34080 Montpellier, FrANCE 